\title{
Telangiectasias perimaculares idiopáticas
}

$\mathbf{T}$

Telangiectasias perimaculares idiopáticas são também conhecidas como telangiectasias retinianas juxtafoveolares ou telangiectasias parafoveais ou perifoveais. Clinicamente, se caracterizam por conjunto de alterações telangiectásicas capilares, perimaculares, uni ou bilaterais e não associadas a doenças sistêmicas. Foram descritas inicialmente por Gass e Oyakawa ${ }^{(1)}$ baseados nos exames clínico e angiofluoresceinográfico. Classificaram-nas em quatro grupos. Grupo 1 - Sexo masculino, envolvimento uniocular, exsudação lipídica intrarretiniana e telangiectasias confinadas a metade temporal da área juxtafoveolar; Grupo 2 - Comumente sexo masculino, com áreas simétricas de telangiectasias afetando a metade temporal das áreas juxtafoveolares e discreta exsudação intrarretiniana; Grupo 3 - Ambos os sexos, com lesões simétricas de todo leito capilar parafoveolar e mínima exsudação; Grupo 4 - Um caso de telangiectasia com alterações oclusivas perifoveolares e palidez de papila de caráter familiar. O prognóstico visual nos grupos 1, 2 e 3 é relativamente bom. A fotocoagulação pode ser útil no tratamento dos pacientes do grupo 1.

Onze anos depois, Gass e Blodi( ${ }^{(2)}$ reviram e atualizaram a classificação em três grupos: 1,2 e 3, com subgrupos A e B nos grupos 1 e 2 baseados em diferenças clínicas e demográficas.

Grupo 1A - Telangiectasias parafoveolares unilaterais congênitas caracterizadas por capilares telangiectásicos, ocupando área de 1 a 2 diâmetros papilares na metade temporal da mácula. Podem ocorrer exsudatos lipídicos nas margens da área de telangiectasias tomando forma de retinite circinata. Idade média de 35 anos e a queda da acuidade visual (AV) (20/25 a 20/40) é devido ao edema macular cistóide e exsudação. Evolução variável podendo manter boa AV por muitos anos. (Figura 1). A fotocoagulação pode ser útil para melhorar ou preservar a AV central. Diagnóstico diferencial com sequelas de retinopatia diabética, retinopatia da irradiação, doença de Eales, anemia falciforme, síndrome isquêmica ocular por oclusão da carótida, doenças inflamatórias adquiridas e distrofias tapetoretinianas.

Grupo 1B - Telangiectasias capilares focais juxtafoveolares idiopáticas com pequena área de capilares incompetentes confinada duas horas ou menos na borda da zona avascular da mácula. Pode haver exsudatos amarelos. AV 20/25 ou melhor. É incerto se é uma lesão adquirida ou representa uma forma leve de telangiectasia congênita (Figuras 1, 2 e 3).

Grupo 2A - Telangiectasias capilares idiopáticas adquiridas parafoveolares, com espessamento da retina temporal à fóvea, podendo estar associadas a "veias em ângulo reto", placas de hiperplasia de epitélio pigmentado

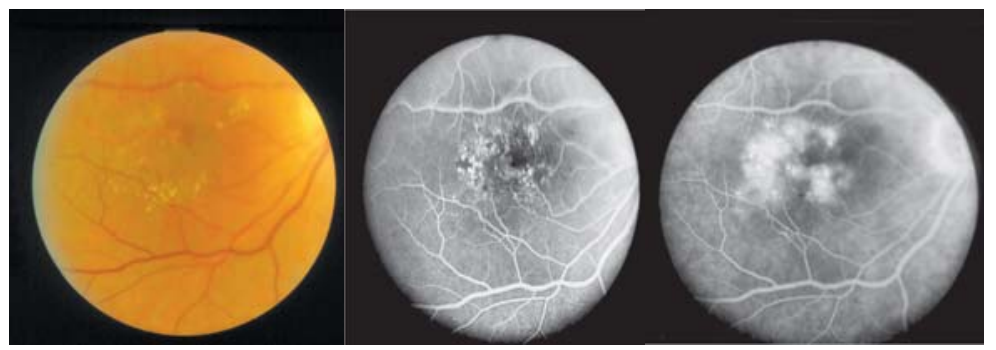

Figura 1

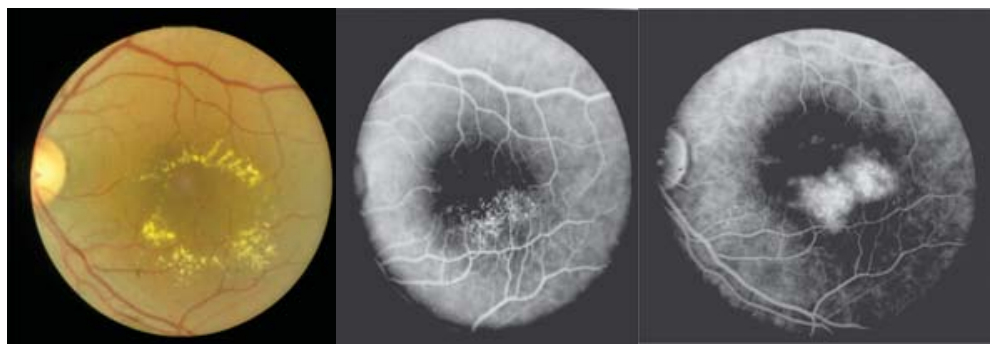

Figuras 1, 2 e 3: Exemplos de telangiectasias idiopáticas juxtafoveais com exsudação lipídica, edema e permeabilidade vascular alterada visíveis na fluoresceinografia

Figura 2

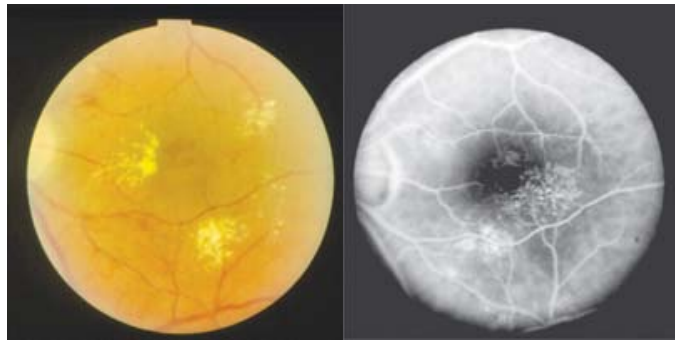

Figura 3 
da retina, depósitos cristalinos, e eventualmente neovascularização sub-retiniana. Idade 50-60 anos, ambos os sexos, lesões simétricas, um disco de papila de diâmetro, ocupando metade temporal da mácula ou toda área macular. Edema e exsudação mínimos (Figuras 4, 5 e 6). Pode ocorrer em fase mais tardia, neovascularização de coróide associada a hemorragias ou exsudatos (Figura 7).

Grupo 2B - Telangiectasias capilares ocultas juvenis e familiares juxtafoveolares. Semelhantes a 2A sem evidências de "vênulas" anguladas, depósitos ou acúmulos pigmentares.

Grupo 3A - Telangiectasias capilares oclusivas com perda visual devido oclusão de capilares perifoveais, ocorrendo na velhice associada a várias doenças sistêmicas como policitemia, hipoglicemia, colite ulcerativa, mieloma múltiplo e leucemia linfática. São lesões semelhantes àquelas que ocorrem na anemia falciforme, retinopatia diabética e retinopatia da irradiação. A perda visual pode ser súbita com retina central de aspecto isquêmico.

Grupo 3B - Mesmas características do Grupo 3A associado à vasculopatia do sistema nervoso central.

Treze anos após, Yannuzzi et al. ${ }^{(3)}$ reviram o assunto e por meio de novas observações baseadas em achados clínicos, fluoresceinográficos, tomografia de coerência óptica e dados demográficos, propuseram nova classificação mais simples em que separavam as telangiectasias maculares idiopáticas em dois grupos: o tipo I - telangiectasias aneurismáticas tipo Coats e o tipo II - telangiectasias perifoveais. O tipo III de Gass e $\mathrm{Blodi}^{(2)}$ foi desconsiderado pela sua raridade e ser a não perfusão capilar como fator mais importante ao invés das telangiectasias maculares como primeira anormalidade.

As telangiectasias tipo I (espectro da doença de Coats) - São telangiectasias aneurismáticas e englobam os

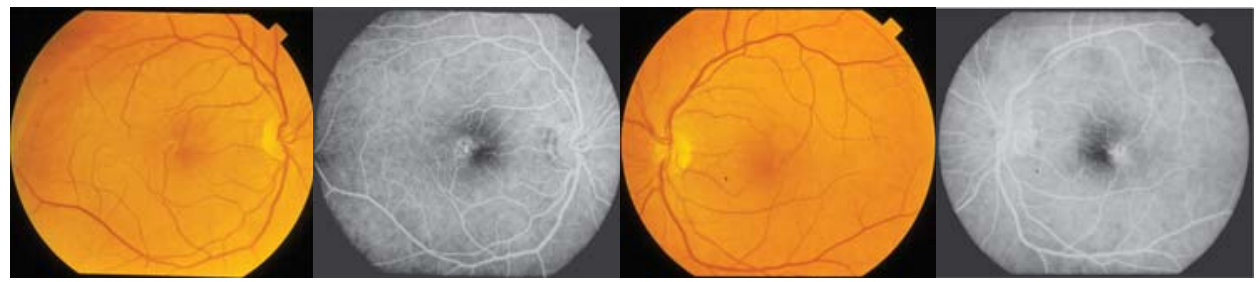

Figura 4 : Telangiectasias parafoveais com vasos angulados

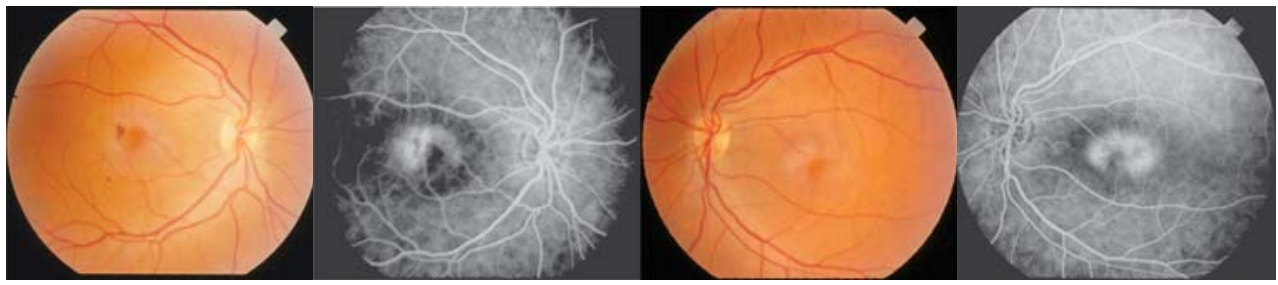

Figura 5: Telangiectasias parafoveais com vasos angulados e hiperplasia do epitélio pigmentar

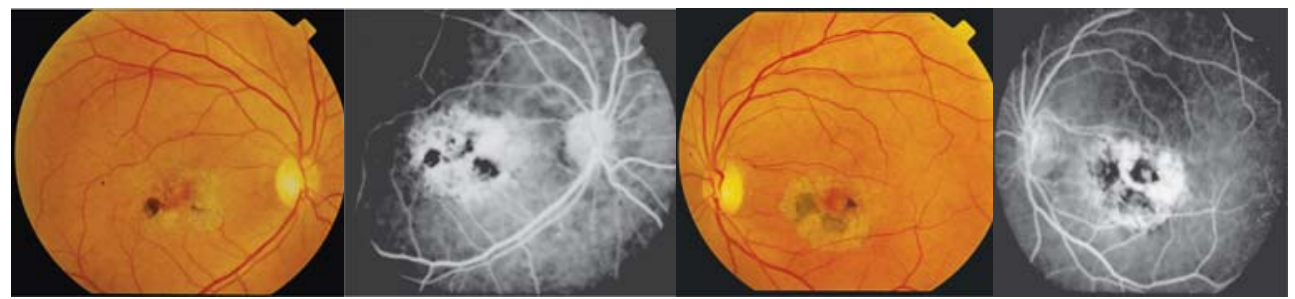

Figura 6: Proliferação neovascular e pigmentar intrarretiniano com vasos angulados
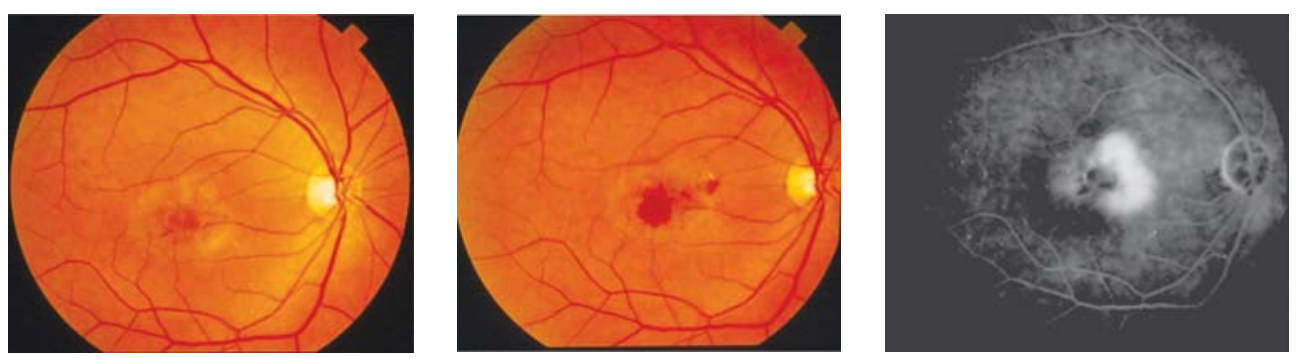

Figura 7: Neovascularização coroidiana hemorrágica, complicação de telangiectasia tipo 2A 
grupos 1A e 1B de Gass e Blodi (2) e omite a diferenciação de 2 horas de relógio do envolvimento juxtafoveal. Ocorrem mais nos homens, unilaterais comumente associadas à exsudação lipídica. Não se observam deposição de cristais, hiperplasia do epitélio pigmentário ou sinais de neovascularização intrarretiniana. Situa-se dentro das anormalidades da doença de Coats, porém perimaculares, podendo ocorrer também na periferia média da retina. Na tomografia de coerência óptica se observam edema macular e alterações císticas na fóvea. O edema é evidente clinica e angiofluoresceinograficamente. Descolamento plano também pode ocorrer detectado pelo OCT. É um processo transudativo através das telangiectasias e capilares incompetentes. Tripathi e Ashton ${ }^{(4)}$ estudaram a histopatologia da doença de Coats por microscopia eletrônica e chegaram a conclusão que a anormalidade fundamental reside no endotélio capilar ou venoso que causa alteração da permeabilidade endotelial com deposição de plasma na parede vascular. Seguem-se rotura gradual da parede vascular com deposição de soro na retina levando a edema e passagem de moléculas lipoprotéicas para a retina causando depósitos de exsudatos lipoproteicos (Figuras 1,2 e 3$)$.

Tipo II - Telangiectasias perifoveais bilaterais, ambos os sexos, com idade média de 59 anos com perda da transparência da retina, pequenos vasos telangiectásicos, vazamento na fluoresceinografia, aparência cística na fóvea sem vazamento intrarretiniano. As veias anguladas podem ser de natureza arteriolar ou venosa. Podem ocorrer anastomoses intrarretinianas e sub-retinianas formando um tecido de proliferação vascular intra ou subretiniana que podem se ligar aos vasos angulados.

A neovascularização sub-retiniana (NVSR) não está necessariamente associada com hiperplasia do epitélio pigmentar (EPR) e pode estar envolvida num envelope de hiperplasia do EPR. Este mostra aspecto sadio, diferente do EPR da degeneração macular relacionada à idade (DMRI). Raramente ocorre descolamento do EPR.

Neste grupo o defeito primário localiza-se nas células de Müller ou células neurais. As células de Müller tem ação crítica na função do endotélio capilar e no metabolismo das células vizinhas. A degeneração destas células de Müller pode causar degeneração endotelial com rotura da barreira hematorretiniana nos capilares parafoveais causando edema não acentuado. As telangiectasias são secundárias e ocorrem no plexo vascular profundo na região temporal juxtafoveal. Conforme sua progressão envolve o plexo capilar superficial e progride anteriormente, posterior e circunferencialmente.

Nas fases mais avançadas o processo vasogênico se estende sob a retina, formando descolamentos e anastomoses retino-retinianas. A progressão deste grupo é definida em 5 estágios:

Estágio 1 - Capilares telangiectásicos profundos não proliferativos. Ocorrem do plexo capilar profundo na área temporal juxtafoveal.

Estágio 2 - Proliferação telangiectásica no plexo capilar interno se anastomosando livremente com o plexo capilar profundo. Ocorre também perda de pigmento lúteo na fóvea. Com o evoluir da doença, o complexo superficial capilar interno é envolvido aparecendo com destaque na fluoresceinografia.

Estágio 3 - Extensão da vasogênese direcionada abaixo da limitante interna. Ocorre também extensão do complexo vascular através da retina e centripetamente em direção a região perifoveal.

Ocorre uma contração e distorção da limitante e sublimitante interna detectados pela fluoresceinografia e tomografia de coerência óptica. Colateralmente pode ocorrer hiperplasia do EPR devido aos estímulos dos vasos profundos direcionados a ele (Figuras 5 e 6).

Cristais podem estar presentes na interface vitreorretiniana, mais evidentes na região temporal e juxtafoveal da mácula. Acredita-se que são processos de mineralização das bases das células de Müller a despeito de não haver confirmação histopatológica. No curso do processo vasogênico, alterações vasculares estimulam a formação de alças vasculares entre arteríolas e vênulas em direção a mácula. Acredita-se ser devido à tentativa de circulação vicariante diante de estímulo de transudação sérica aumentada.

Estágio 4 - Proliferativa - Evoluindo o processo vasogênico pode ocorrer para neovascularização subretiniana que é indistinguível da neovascularização coroidiana. É um processo de proliferação vascular intrarretiniana, semelhante ao RAP (Retinal Angiomatous Proliferation) ${ }^{(5,6)}$. Nessa fase, a despeito da proliferação vascular, o EPR mantém-se intacto (Figura 6). Pode ocorrer complicação de neovascularização coroidiana acompanhada de hemorragias ou exsudatos.

Estágio 5 - Proliferação fibrovascular que se estende pelo espaço sub-retiniano na área foveolar central. Anastomoses retinianas podem ser observadas sem envolvimento da circulação da coróide que também pode ocorrer, porém raramente (Figura 7).

Diagnóstico diferencial deve ser feito com as telangiectasias secundárias a doenças vasculares da retina como oclusões venosas, retinopatias diabética, da irradiação, anemia falciforme, hipertensão arterial, síndrome ocular isquêmica por obstrução da carótida, policitemia, hemangioma capilar da retina, degenerações tapetoretinianas e doenças inflamatórias adquiridas.

Tratamento - Opções para tratamento das telangiectasias perifoveais tipos 1 e 2 incluem fotocoagulação ${ }^{(7)}$, injeções intravítreas de esteróides ${ }^{(8-10)}$, agentes antiVEGF ${ }^{(11-13)}$, terapia fotodinâmica ${ }^{(14-16)}$ e termoterapia ${ }^{(17)}$.

A estratégia do tratamento depende da acuidade visual, fluoresceinografia, tomografia de coerência óptica e biomicroscopia da retina. Na fotocoagulação( ${ }^{(7)}$ o foco são as telangiectasias geradoras de transudação e distantes da fóvea pelo menos 500 micra. 
Evidentemente são selecionadas as miras, intensidade e tempo de exposição adequada para os mínimos efeitos colaterais do disparo. Essa indicação é mais comum nas telangiectasias tipo 1 (Mactel 1).

As injeções intravítreas de esteroides ${ }^{(8-10)}$ demonstraram melhora do edema macular melhorando a permeabilidade vascular das telangiectasias. Todavia o efeito é passageiro com recidiva do edema em 3 a 6 meses e sem melhora da acuidade visual. Como complicações se observam em alguns casos elevação da pressão intraocular e formação de catarata.

As injeções intravítreas de agentes antiVEGF ${ }^{(11-13)}$ como bevacizumab e ranibizumab (anticorpos monoclonais humanizados) demonstraram melhora do resultado visual e redução do vazamento do contraste no edema macular. Novas pesquisas estão acontecendo para definir a melhor estratégia para as várias fases das doenças.

A termoterapia transpupilar ${ }^{(17)}$ pode ser uma opção útil em tratamento nas neovascularizações sub-retinianas.

Vários estudos pelo Mactel Study Group estão em andamento procurando correlacionar a função retiniana com a tomografia de coerência óptica, autofluorescência, microperimetria, acuidade visual, metamorfopsia e tratamento ${ }^{(18-20)}$.

Em verdade, essas duas formas de telangiectasias adquiridas foram agrupadas pelos autores, respeitando-se somente a topografia perimacular das mesmas. A fisiopatogenia, quadro clínico, evolução, prognóstico e tratamento de ambos os tipos são diferentes.

Comumente, as classificações nosológicas respeitam as fisiopatogenias e quadro clínico das doenças e, neste caso, a dissociação futura dessas duas entidades patológicas poderá ocorrer com o intuito da melhoria do estudo, ensino e tratamento das telangiectasias maculares (congênitas e adquiridas).

Faculdade de Medicina da Universidade de São Paulo - USP - São Paulo (SP), Brasil

\section{Suel Abujamra}

\section{REFERÊNCIAS}

1. Gass JD, Oyakawa RT. Idiopathic juxtafoveolar retinal telangiectasis. Arch Ophthalmol. 1982;100(5):769-80.

2. Gass JD, Blodi BA. Idiopathic juxtafovealar retinal telangiectasis. Update of classification and follow-up study. Ophthalmology. 1993;100(10):1536-46.

3. Yannuzzi LA, Bardal AM, Freund KB, Chen KJ, Eandi CM, Blodi B. C. Idiopathic macular telangiectasia. Arch Ophthalmol. 2006;124(4):450-60. Comment in Arch Ophthalmol. 2006;124(4):573-4.

4. Tripathi R, Ashton N. Electron microscopical study of Coat's disease. Br J Ophthalmol. 1971;55(5):289-301.

5. Yannuzzi LA, Negrão S, Iida T, Carvalho C, Rodriguez-Coleman H, Slakter J, et al. Retinal angiomatous proliferation in agerelated macular degeneration. Retina. 2001;21(5):416-34

6. Gass JD. Chorioretinal anastomosis probably occurs infrequently in type 2A idiopathic juxtafoveolar retinal telangiectasis. Arch Ophthalmol. 2003;121(9):1345-6. Comment on Arch Ophthalmol. 2002;120(3):320-4.

7. Stoffelns BM, Schoepfer K, Kramann C. [Idiopathic macular telangiectasia - follow-up with and without laser photocoagulation]. Klin Monbl Augenheilkd. 2010;227(4):252-6. German.

8. Alldredge CD, Garretson BR. Intravitreal triamcinolone for the treatment of idiopathic juxtafoveal telangiectasis. Retina. 2003;23(1):113-6.

9. Martinez JA. Intravitreal triamcinolone acetonide for bilateral acquired parafoveal telangiectasis. Arch Ophthalmol. 2003;121(11):1658-9.

10. Wu L, Evans T, Arévalo JF, Berrocal MH, Rodríguez FJ, Hsu M, Sánchez JG. Long-term effect of intravitreal triamcinolone in the nonproliferative stage of type II idiopathic parafoveal telangiectasia. Retina. 2008;28(2):314-9.

11. Moon SJ, Berger AS, Tolentino MJ, Misch DM. Intravitreal bevacizumab for macular edema from idiopathic juxtafoveal retinal telangiectasis. Ophthalmic Surg Lasers Imaging. 2007;38(2):164-6.

12. Charbel Issa P, Finger RP, Holz FG, Scholl HP. Eighteen-month follow-up of intravitreal bevacizumab in type 2 idiopathic macular telangiectasia. Br J Ophthalmol. 2008;92(7):941-5.

13. Vianna RN, Squeri G, Turquetti R, Brasil OF, Burnier MN Jr. Intravitreal pegaptanib reduces fluorescein leakage in idiopathic parafoveal telangiectasis. Can J Ophthalmol. 2008;43(4):492-3.

14. Potter MJ, Szabo SM, Sarraf D, Michels R, Schmidt-Erfurth U. Photodynamic therapy for subretinal neovascularization in type 2A idiopathic juxtafoveolar telangiectasis. Can J Ophthalmol. 2006;41(1):34-7.

15. Shanmugam MP, Agarwal M. RPE atrophy following photodynamic therapy in type $2 \mathrm{~A}$ idiopathic parafoveal telangiectasis. Indian J Ophthalmol. 2005;53(1):61-3.

16. Hussain N, Das T, Sumasri K, Ram LS. Bilateral sequential photodynamic therapy for sub-retinal neovascularization with type 2A parafoveal telangiectasis. Am J Ophthalmol. 2005;140(2):333-5.

17. Shukla D, Singh J, Kolluru CM, Kim R, Namperumalsamy P. Transpupillary thermotherapy for subfoveal neovascularization secondary to group 2A idiopathic juxtafoveolar telangiectasis. Am J Ophthalmol. 2004;138(1):147-9. Comment in Am J Ophthalmol. 2005;139(3):577-8; author reply 578.

18. Clemons TE, Gillies MC, Chew EY, Bird AC, Peto T, Figueroa M, Harrington MW; Macular Telangiectasia Research Group. The National Eye Institute Visual Function Questionnaire in the Macular Telangiectasia (MacTel) Project. Invest Ophthalmol Vis Sci. 2008;49(10):4340-6.

19. Charbel Issa P, Helb HM, Holz FG, Scholl HP; MacTel Study Group. Correlation of macular function with retinal thickness in nonproliferative type 2 idiopathic macular telangiectasia. Am J Ophthalmol. 2008;145(1):169-75.

20. Wong WT, Forooghian F, Majumdar Z, Bonner RF, Cunningham D, Chew EY. Fundus autofluorescence in type 2 idiopathic macular telangiectasia: correlation with optical coherence tomography and microperimetry. Am J Ophthalmol. 2009;148(4):573-83. 